\title{
Research on Audio and Video Synchronization Algorithm Based on AVI Format
}

\author{
Min Zhang ${ }^{1, a}$, Yu-Hua $\mathrm{Wu}^{1, b}, \mathrm{Ji} \mathrm{Li}^{1, \mathrm{c}}$, Shi-Jun $\mathrm{Li}^{\mathrm{d}, *}$ \\ ${ }^{1}$ Jilin Agricultural University, Changchun 130118, Jilin, China \\ a1165339875@qq.com, bawuyuhua@163.com, '35485328@qq.com, ${ }^{\mathrm{d}}$ Isj0883@sina.com \\ *Shi-Jun LI
}

Keywords: AVI format, Decode, Synchronization, Video, Audio.

\begin{abstract}
This paper is to synchronize the AVI media file on the video player. It is that audio playback clock is used as synchronous clock. It is using FFmpeg to decode AVI video files to get audio and video data, and calculate the audio playback clock to audio playback clock as a reference clock to load video playback, to ensure the audio and video synchronization play, there will be no information disorder. Finally the experimental shows that the video player can be a good decoding AVI video files, and can guarantee audio and video synchronization play.
\end{abstract}

\section{Introduction}

With the development of Internet network technology, remote video conferencing, online live, on demand and other multimedia services have been widely used in all aspects of life. However, in the process of network transmission of video information, due to network jitter, packet loss and other factors [1], resulting in the player to accept and play the video audio and video when the phenomenon of synchronization, affecting the user experience.

In order to solve the audio and video synchronization problems and improve the user experience. There are many scholars to provide a lot of different ways to achieve video and audio synchronization. These audio and video synchronization can be divided into embedded synchronous and non-embedded synchronization mode. Non-embedded synchronization method [2] through the use of timestamp method to achieve audio and video synchronization; [3] by sending the establishment of the reference clock, so that after the information sent to the receiver, in the acceptance of the reference time stamp Audio and video synchronization. In [4], audio and video synchronization is achieved by comparing the timestamps to the audio and video at the transmitting end and by comparing the relative timestamps at the receiving end. In the embedded synchronization method, the audio data synchronization is realized by using the optimal matching point of 1/4 pixel in the motion estimation of H.264 to realize the audio and video synchronization[5]. In [6], the audio data is embedded in the inter-frame prediction process To synchronize.

Based on the above analysis, embedded and non embedded synchronization algorithm has high complexity and complex operation. In this paper is to using FFmpeg decoding AVI format video files, while the use of audio playback time for the reference clock synchronization mode, to achieve the AVI file format for analysis and synchronization playback player.

\section{AVI Format}

The AVI video file format is a technical standard developed by Microsoft that can be used extensively for digital video compression. According to the technical standards defined, it is possible to extract the AVI video file from the digital storage device to obtain the data stream and perform the frame-by-frame decomposition [7].

Audio Video Interleaved(AVI) is audio and video data intertwined storage of a video file format, a container format, the Windows system is the most commonly used media file format. According to the AVI file format standard, it is not only contain a number of different types of media stream 
information, but also allows only a single video stream information or a single audio stream information.

The AVI file is mainly organized in units of data blocks, each data block through a four-character code FOURCC (four-character code) to represent the type of data, such as 'AVI', 'LIST' and other types, followed by 4-byte integer represents the size of the current block, and the remainder is the actually valid multimedia data [9]. AVI file format shown in Figure 1.

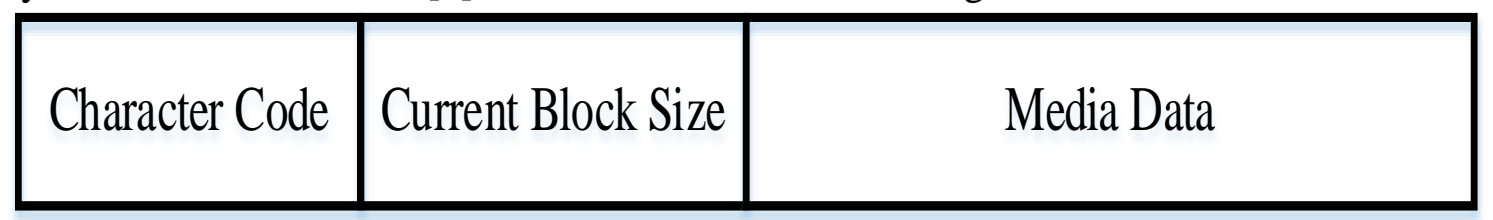

Fig. 1 AVI file format

\section{FFmpeg decoding and SDL display}

\subsection{Decoding process Based on FFmpeg}

FFmpeg is a free software project that development under the Linux system with $\mathrm{C}$ language for multimedia processing. At the same time in Windows, Mac and other operating systems to compile and use, with good cross-platform features. It not only has a rich audio and video codec library, but also contains the recording, video file protocol format analysis and other related functions. FFmpeg consists of eight core libraries respectively: codec file, media format library file, device operation related library file, filter processing library file, image operation and color format conversion library file for processing audio sampling data Library file.

FFmpeg decode audio and video process: use av_register_all () function to register all components of FFmpeg, to ensure that FFmpeg can start work; through avformat_open_input () function to open AVI multimedia files; avformat_find_stream_info () function to obtain AVI file audio stream compression data and video Stream compression data; through the avcodec_find_decoder () function to find the audio stream data and video stream data corresponding to the decoder information, and then decode the audio and video data, and finally get the original audio data (PCM) and the original video data (YUV). FFmpeg decoding process shown in Figure 2.

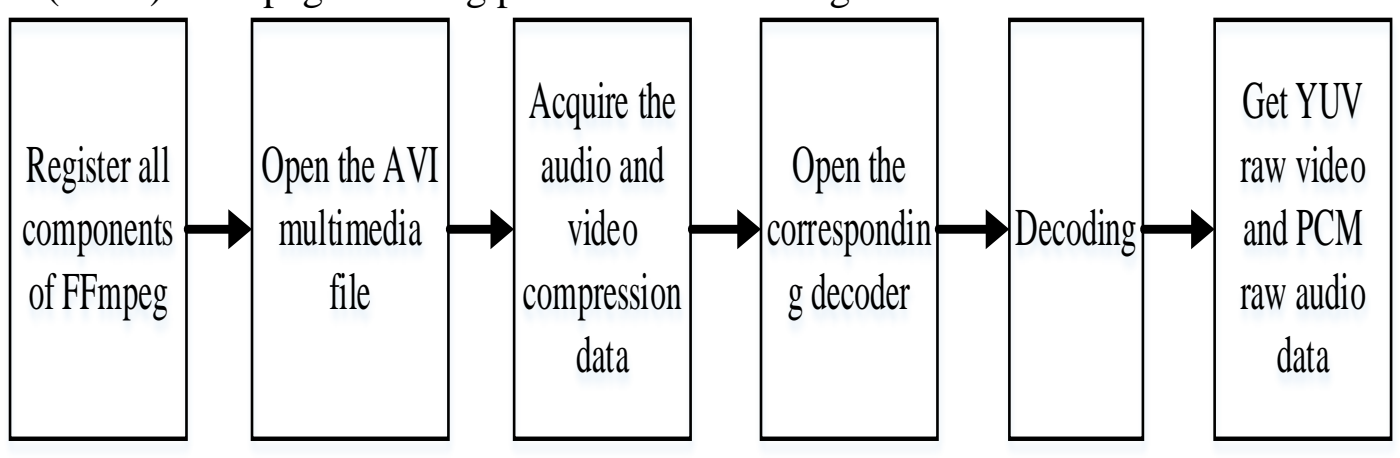

Fig. 2 FFmpeg decoding process

\subsection{SDL Display}

Simple DirectMedia Layer(SDL) SDL is a cross-platform open source multimedia display library that development with C language. It is Mainly used for games, simulators, multimedia player applications and other aspects of the development. SDL itself provides a wealth of audio and video processing API function, can be well into the $\mathrm{C}++$, Java and other advanced programming language for the corresponding development, very convenient.

The use of SDL to realize the audio playback and video display, first use SDL_Init () function to initialize the SDL library; using SDL_CreateWindow () to create a display window for displaying a video image size; the actual use of SDL_Rect structure definition video screen in the display window size; through update the video data from the decoding display on the screen, SDL video display process as shown in Figure 3. Through the SDL_OpenAudio () function to open the audio playback 
device, and then use the audio parameters related to the SDL_AudioSpec structure defined in the audio sampling related information to the audio device for playback.

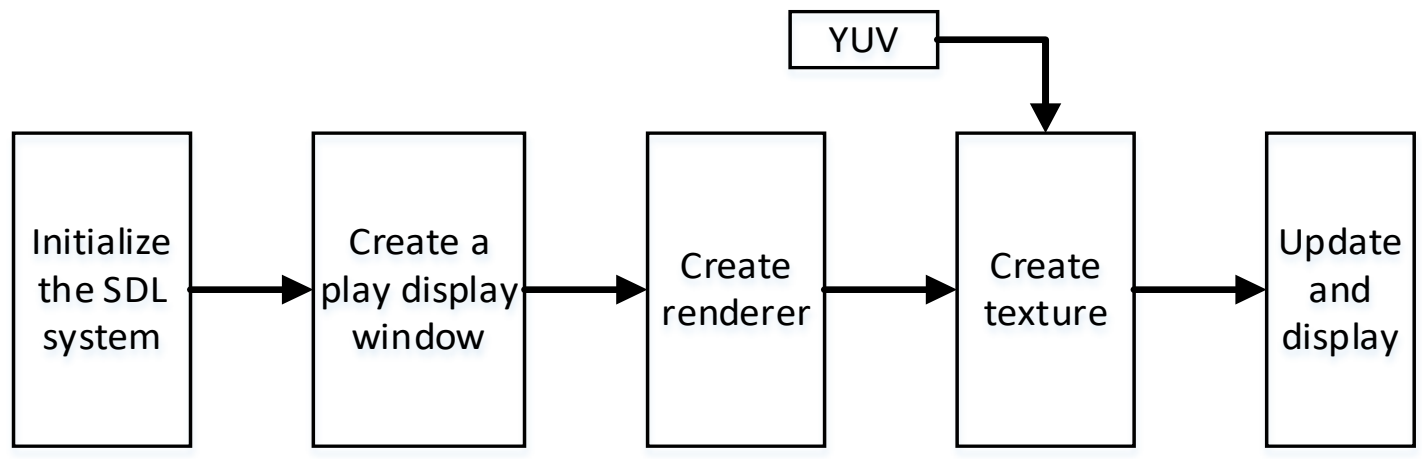

Fig. 3 SDL display process

\section{Audio and Video Synchronization}

In this paper, audio playback time based on the synchronization mode to achieve audio and video synchronization. The audio playback time is the reference clock. When the difference between the video playback clock and the reference time is between $-90 \mathrm{~ms}$ and $20 \mathrm{~ms}$ of ITU-R BT.1359-1[9], it is considered that the audio and video are synchronous. If the video is playing faster, start the delay to synchronize the clock. If the video is slow to play faster to speed up the video to achieve synchronization. Specific implementation process shown in Figure 3.

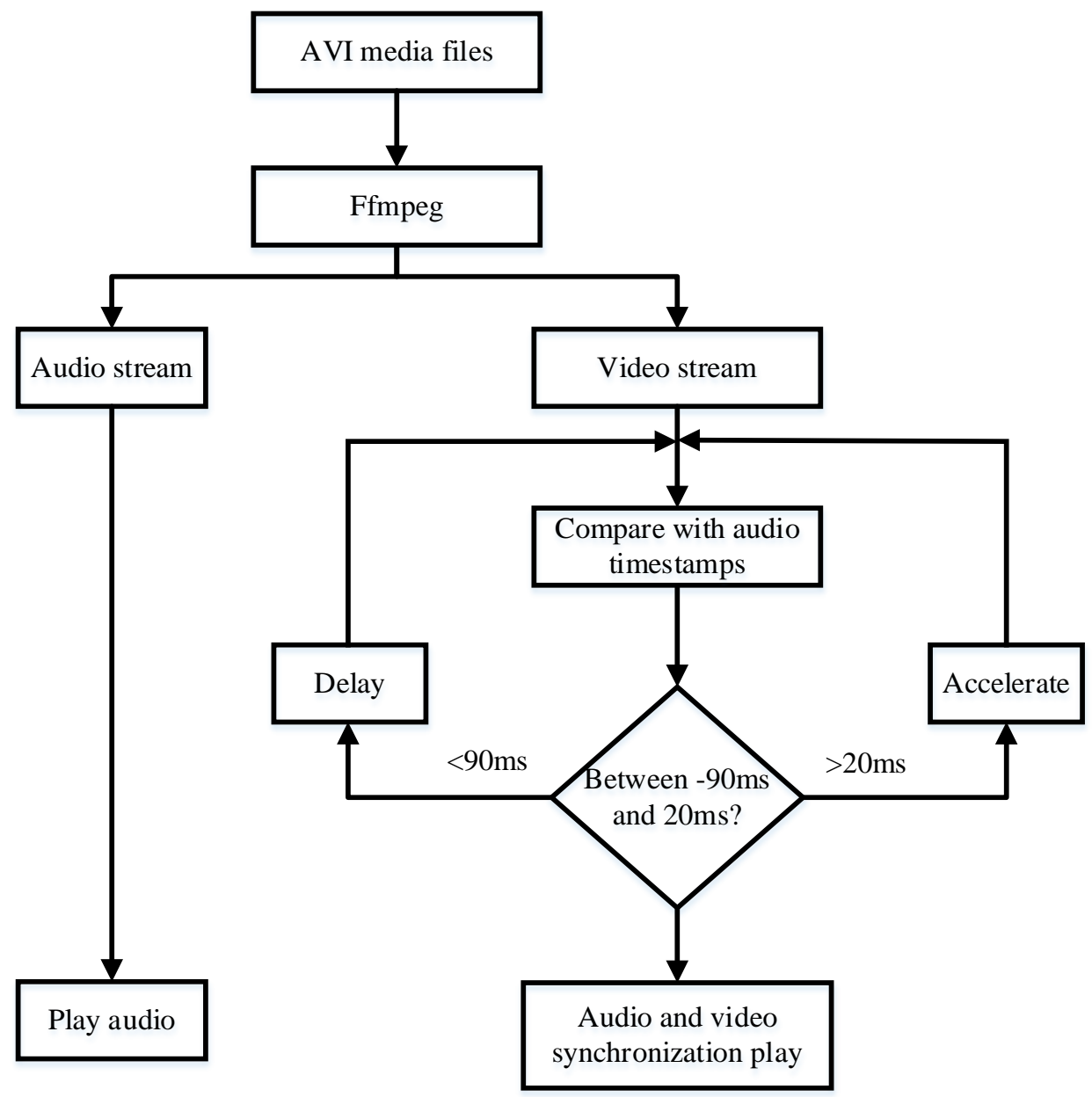

Fig. 4 Synchronize the playback process 


\section{Conclusions}

This paper describes the FFmpeg decoding process and SDL audio and video playback display process, and by audio playback clock as the reference clock to synchronize the way to play video to ensure the synchronization of audio and video playback, audio and video information will not appear disorder phenomenon.

\section{Acknowledgments}

The authors wish to express their gratitude to the projects: Research on Audio and Video Synchronization Algorithm Based on AVI Format from Education Department of Jilin Province, Jilin Province Economic Structural Adjustment Leading Fund Special Project (No. 2014Y108) and Changchun City Science and Technology Plan Project (No. 14nk029), Jilin Province Science and Technology Development Program Funded Project (No. 20160623016TC), Education Department of Jilin Province's $12^{\text {th }}$ Five-Year Science and Technology Research Project (2015175), for their generous support of this work. At the same time, the other authors also thank the corresponding author, Shijun LI, for his assistance in submitting this paper.

\section{References}

[1] SHI Meiqiang, LI Bing, XIONG Jun, ZHAO Qilin. Video and Audio Synchronization Compression Method Based on H.264/AVC[J]. Video Engineering, 2009, (10):15-17+27.

[2] EI-Helaly M, Amer A. Synchronization of Processed Audio-video Signals Using Time-stamps[C]//Proceed-ings of International Conference on Image Processing. Washington D. C. USA: IEEE Press, 2007:193-196.

[3] MA Honglei, LIU Er wu, ZHU Jun. Design of Audio and Video Synchronization Algorithm for Asymmetric Wireless Transmission[J]. Computer Simulation, 2016, (02):221-226+479.

[4] LU Zhiwei Research on Audio and Video Synchronous Transmission and Its Realization in Virtual Desktop System[D]. University of Electronic Science and Technology of China, 2015.

[5] LI Xiaoni, CHEN Hexin, CHEN Mianshu, GABBOUJ Moncef. Audio-video synchronous coding based on motion estimation in H.264[J]. Journal of Jilin University(Engineering and Technology Edition), 2012, (05):1321-1326.

[6] LI Xiaoni, CHEN Hexin, SUN Yuan, CHEN Mianshu, LIU Tian. Embedded audio-video synchronization coding based on H .264[J]. Journal of Jilin University(Engineering and Technology Edition), 2011, (05):1475-1479.

[7] LIU Huiwei. Visual C ++ video / audio development practical project case selection[M]. Beijing: People 's Posts and Telecommunications Press, 2004.

[8] CUI Zhihui. Research on Vehicle Detection Method Based on Video Image[D]. Soochow University, 2012.

[9] ITU-R BT. 1359-1. Relative timing of sound and vision for broadcasting[S]. 1998. 